\title{
On the calculation of heat production from open-circuit calorimetric measurements
}

\author{
By J. A. McLEAN \\ Department of Physiology, Hannah Dairy Research Institute, Ayr

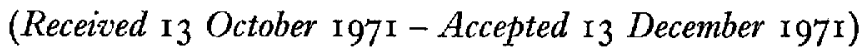

\begin{abstract}
1. The equation used for calculating heat production of ruminant animals is expressed in a new form, convenient for application to open-circuit calorimetry.

2. It is shown that in open-circuit calorimetry the possible crror involved in making the assumption that respiratory quotient is equal to unity is small compared with the possible error involved in neglecting methane production.

3. In open-circuit calorimetry heat production can be predicted with accuracy to within $\pm 2 \%$ solely from the measurement of oxygen concentration and ventilation rate.
\end{abstract}

The Brouwer equation for calculating heat production, in its most recently revised form (Brouwer, 1965 ), is

$$
H=\left(3.866 \times \mathrm{O}_{2}\right)+\left(\mathrm{I} \cdot 200 \times \mathrm{CO}_{2}\right)-\left(0.5 \mathrm{I} 8 \times \mathrm{CH}_{4}\right)-(\mathrm{I} \cdot 43 \mathrm{I} \times \mathrm{N}),
$$

where $\mathrm{H}=$ heat production (kcal), $\mathrm{O}_{2}, \mathrm{CO}_{2}$ and $\mathrm{CH}_{4}$ represent volumes of oxygen consumed and of carbon dioxide and methane produced (l) and $\mathrm{N}$ is the quantity of urinary nitrogen excreted $(\mathrm{g})$.

In closed-circuit calorimetry direct measurement is made of the consumption of oxygen and production of carbon dioxide and, according to the Brouwer equation, the terms containing these measurements contribute approximately 75 and $25 \%$ respectively to the total heat production. The other two terms each contribute about I $\%$.

In open-circuit calorimetry the direct measurements are ventilation rate and the composition of inlet and outlet air, and it is customary to use these values to calculate the quantities of gases consumed or produced. This involves evaluating a 'volume correction factor' (VCF). This paper shows that if the VCF is written in a suitable form its substitution into the Brouwer equation results in considerable simplification of the calculation procedure. It also shows that further approximations permitting the omission of all gas measurements except that of oxygen may frequently be justified.

If the volumes of inlet and outlet air in an open-circuit calorimetric system (whether it be an animal chamber, head cage or face mask) are $V_{i}$ and $V_{o}$ respectively, and the gas concentrations are as shown in Table $\mathrm{I}$, then the VCF is given by

$$
\begin{aligned}
\mathrm{VCF} & =\frac{V_{i}}{V_{o}}=\frac{\mathrm{I}-x_{o}-y_{o}-z_{o}}{\mathrm{I}-x_{i}-y_{i}-z_{i}} \\
& =\frac{\mathrm{I}-x_{i}-y_{i}-z_{i}+x_{i}+y_{i}+z_{i}-x_{o}-y_{o}-z_{o}}{\mathrm{I}-x_{i}-y_{i}-z_{i}} \\
& =\mathrm{I}+\frac{\mathrm{I}}{N_{i}}(X-Y-Z) .
\end{aligned}
$$


Table I. Symbols used for gas concentrations in inlet and outlet air

$\begin{array}{lccc}\text { Gas } & \text { Inlet air } & \text { Outlet air } & \text { Difference } \\ \mathrm{O}_{2} & x_{i} & x_{o} & X=\left(x_{i}-x_{o}\right) \\ \mathrm{CO}_{2} & y_{i} & y_{0} & Y=\left(y_{o}-y_{i}\right) \\ \mathrm{CH}_{4} & z_{i} & z_{o} & Z=\left(z_{0}-z_{i}\right) \\ \mathrm{N}_{2} & \left(\mathrm{I}-x_{i}-y_{i}-z_{i}\right)=N_{i} & \left(\mathrm{I}-x_{0}-y_{0}-z_{o}\right) & \end{array}$

Oxygen consumption may therefore be expressed as

similarly

$$
\begin{aligned}
\mathrm{O}_{2} & =V_{i} x_{i}-V_{o} x_{o} \\
& =V_{o}\left[X+\frac{x_{i}}{N_{i}}(X-Y-Z)\right]
\end{aligned}
$$

and

$$
\mathrm{CO}_{2}=V_{o}\left[Y-\frac{y_{i}}{N_{i}}(X-Y-Z)\right]
$$

$$
\mathrm{CH}_{4}=V_{o}\left[Z-\frac{z_{i}}{N_{i}}(X-Y-Z)\right] .
$$

Substituting for $\mathrm{O}_{2}, \mathrm{CO}_{2}$ and $\mathrm{CH}_{4}$ in the Brouwer equation and collecting terms in $X, Y$ and $Z$ gives the general equation

$$
H=V_{o}[(3 \cdot 866+\mu) X+(\mathrm{I} \cdot 200-\mu) Y-(0 \cdot 5 \mathrm{I} 8+\mu) Z]-\mathrm{I} \cdot 43^{\mathrm{I}} N
$$

where $\mu=\left(3 \cdot 866 x_{i}-\mathrm{I} \cdot 200 y_{i}+0.5 \mathrm{I} 8 z_{i}\right) / N_{i}$.

The value of $\mu$ depends only on the composition of inlet air. In many instances inlet air is atmospheric air $\left(x_{i}=0.2095, y_{i}=0.0003, z_{i}=0\right)$ (Weast, 1970-I) for which $\mu=\mathrm{I} \cdot 025$; hence

$$
H=V_{o}[4 \cdot 89 \mathrm{I} X+0 \cdot 175 Y-\mathrm{I} \cdot 543 Z]-\mathrm{I} \cdot 43 \mathrm{I} N .
$$

This equation contains only quantities which are direct measurements in opencircuit calorimetry and it is therefore more convenient to use than the Brouwer equation in its usual form. The contribution of the carbon dioxide term (containing $Y$ ) to the heat production is here only of the order of $3 \%$, very much less than in the Brouwer equation. Also it is roughly equal but of opposite sign to the methane term (containing $Z$ ), since the rate of $\mathrm{CO}_{2}$ production in ruminants is ten to fifteen times that of $\mathrm{CH}_{4}$ production. A reasonably good approximation is therefore obtained by omitting all except the oxygen term.

An alternative way of writing the general equation is:

$$
H=V_{0}[5 \cdot 066 X-(\mathrm{I} \cdot 200-\mu)(\mathrm{r}-[Y / X]) X-(0 \cdot 5 \mathrm{I} 8+\mu) Z]-\mathrm{r} \cdot 43 \mathrm{I} N .
$$

The quantity $Y \mid X$ is an approximate measure of the respiratory quotient and it may be shown that its value always lies between that of respiratory quotient and unity (see p. 600).

The value of $\mu$ depends only on inlet air composition, and under practical conditions will always lie within $\mu=\mathrm{r} \cdot 013 \pm 0.013$. The greatest possible magnitude of the 
second term within the square parentheses is therefore $0.04 X$, or $\frac{1}{12}$ th part of the first term, and it will usually be much less. For most purposes it may be neglected; hence

$$
H=V_{o}[5 \cdot 066 X-\mathrm{I} \cdot 53 Z]-\mathrm{I} \cdot 43 \mathrm{I} N \text {. }
$$

This equation, which is always accurate to within less than $\pm 0.8 \%$, eliminates the need for the measurement of carbon dioxide concentration and emphasizes the need for the measurement of methane concentration, which is in fact the more significant factor in open-circuit calorimetry, contributing approximately $3 \%$ to the accuracy of the measurement of heat production.

For greatest possible accuracy it is of course necessary to measure oxygen, carbon dioxide and methane concentrations as well as urinary nitrogen excretion rate. But for approximate measurements by open-circuit calorimetry it is preferable to measure oxygen and methane concentrations and ignore carbon dioxide than to measure oxygen and carbon dioxide concentrations and ignore methane, as is frequently done.

Further simplification may be made in open-circuit calorimetry by making assumptions regarding methane production and urinary nitrogen excretion, rather than by ignoring these terms altogether. K. L. Blaxter (personal communication) found from ninety 5 - $\mathrm{d}$ experiments on animals that methane production/l oxygen consumed averaged $0.084( \pm 0.020 \mathrm{sD}) \mathrm{l}$. In the same series of experiments urinary nitrogen excretion $/ 1$ oxygen consumed was 0.032 ( $\pm 0.010 \mathrm{sD}) \mathrm{g}$. Although these values refer to a specific series of experiments, they cover a wide variety of feeding levels and diets and represent reasonably approximate values to substitute for $Z / X$ and $N / V_{o} X$ in the equation, which then becomes

$$
H=4^{\cdot 8 g} V_{o} X
$$

This equation is similar to that for man derived by Weir (1949) using a different approach. The fortunate coincidence that the terms containing methane and carbon dioxide concentration in the full equation for ruminants are approximately equal and opposite to each other makes the simple equation, containing only oxygen, a better approximation than is that for man. It is estimated that this simple equation for ruminants is accurate to within $\pm 2 \%$ except under severely abnormal feeding conditions.

\section{APPENDIX}

Proof that the value of $Y \mid X$ is close to respiratory quotient $(R)$

Consider an open-circuit system where $\mathrm{O}_{2}$ consumption $=A, \mathrm{CO}_{2}$ production $=A R, \mathrm{CH}_{4}$ production $=A F$. Then the concentrations and volumes are derived as shown in Table 2 . From the gas concentrations in Table 2 it follows that:

$$
\begin{aligned}
X & =x_{i}-\frac{x_{i} V_{i}-A}{V_{i}-A(\mathrm{I}-R-F)} \\
& =\frac{A-x_{i} A(\mathrm{I}-R-F)}{V_{i}-A(\mathrm{I}-R-F)},
\end{aligned}
$$


and

$$
\begin{aligned}
Y & =\frac{A R+y_{i} A(\mathrm{I}-R-F)}{V_{i}-A(\mathrm{I}-R-F)}, \\
\therefore \frac{Y}{X} & =\frac{R+y_{i}(\mathrm{I}-R-F)}{\mathrm{I}-x_{i}(\mathrm{I}-R-F)} .
\end{aligned}
$$

Table 2. Gas concentrations and volumes in open-circuit calorimetry

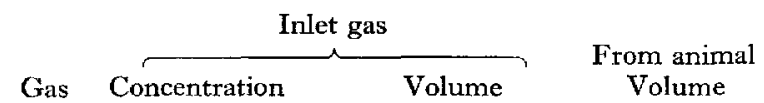

$\mathrm{O}_{2} \quad x_{i}$

$\mathrm{CO}_{2} \quad y_{i} \quad y_{i} V_{i}$

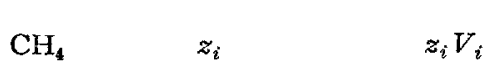

$\begin{array}{ccc}\mathrm{N}_{2} & \mathrm{I}-x_{i}-y_{i}-z_{i} & \left(\mathrm{I}-x_{i}-y_{i}-z_{i}\right) V_{i} \\ \text { Total } & \mathrm{I} & V_{i}\end{array}$

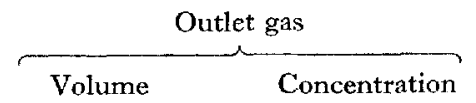

$-A \quad x_{i} V_{i}-A$

$\frac{x_{i} V_{i}-A}{V_{i}-A(\mathrm{I}-R-F)}$

$A R \quad y_{i} V_{i}+A R$

$\frac{y_{i} V_{i}+A R}{V_{i}-A(\mathrm{I}-R-F)}$

$A F$

O

$$
\frac{z_{i} V_{i}+A F}{V_{i}-A(\mathrm{I}-R-F)}
$$

$\left(1-x_{i}-y_{i}-z_{i}\right) V_{i}$

$-A(\mathrm{I}-R-F) \quad V_{i}-A(\mathrm{I}-R-F)$

Using atmospheric air values $\left(x_{i}=0.21, y_{i}=0.0003\right)$ and neglecting methane $(F=0)$ :

$$
\begin{aligned}
\frac{X}{Y} & \bumpeq \frac{R}{\mathrm{I}-0.2 \mathrm{I}(\mathrm{I}-R)}, \\
& \bumpeq R[\mathrm{I}+0.2 \mathrm{I}(\mathrm{I}-R)],
\end{aligned}
$$

when $R=\mathrm{I}, Y / X=\mathrm{I}$. Over the practical range, $\mathrm{I} \cdot 25>R>0.75$, and therefore $\mathrm{I} \cdot \mathrm{I} 9>Y \mid X>0.78$.

$Y / X$ is thus always slightly closer to unity than is $R$.

The author is grateful to Dr K. L. Blaxter for kindly supplying the experimental values for methane: oxygen and urinary nitrogen:oxygen ratios.

\section{REFERENCES}

Brouwer, E. (I965). In Energy Metabolism p. 44I [K. L. Blaxter, editor]. London: Academic Press. Weast, R. C. (editor) (I970-I). The Handbook of Chemistry and Physics 5 Ist ed. Cleveland, Ohio:

The Chemical Rubber Co.

Weir, J. B. de V. (1949). F. Physiol, Lond. ro9, r. 